\title{
Impacto da cefaleia primária na qualidade de vida de policiais militares e sua relação com transtorno mental comum
}

\author{
Impact of primary headache on quality of life in military policemen and \\ its relation to common mental disorder
}

\author{
Lorena Costa Leite', Nahãmi Cruz de Lucena², Marcelo Moraes Valença ${ }^{3}$, Daniella Araújo de Oliveira ${ }^{1}$ \\ 'Departamento de Fisioterapia, Universidade Federal de Pernambuco (UFPE), Recife, PE, Brasil \\ ${ }^{2}$ Departamento de Fisioterapia, Instituto de Medicina Integral Professor Fernando Figueira - Imip, Recife, PE, Brasil \\ ${ }^{3}$ Departamento de Neuropsiquiatria, Universidade Federal de Pernambuco (UFPE), Recife, PE, Brasil \\ Leite LC, Lucena NC, Valença MM, Oliveira DA. Impacto da cefaleia primária na qualidade de vida de policiais \\ militares e sua relação com transtorno mental comum. Headache Medicine. 2013;4(4):96-101
}

\begin{abstract}
RESUMO
Objetivo: Determinar o impacto gerado pela cefaleia primária na qualidade de vida dos policiais militares do Recife e sua relação com a presença de transtornos mentais comuns. Métodos: Trata-se de um estudo transversal, com 211 policiais militares de rua, homens, atualmente da ativa, com idade entre 20 e 50 anos (33,0 0,7 anos). A presença de cefaleia foi avaliada por meio de um questionário baseado no ICHD-II, considerando a presença de cefaleia nos últimos três meses. Para avaliar o impacto causado pela cefaleia foi utilizado o questionário Headache Impact Test-6. Para o rastreamento de transtorno mental comum foi utilizado o questionário Self Reporting Questionnaire-20. A intensidade da dor foi mensurada através da escala numérica da dor. Resultados: 142/211 policiais $(67,3 \%)$ apresentaram cefaleia nos últimos três meses. Destes 57/142 (40,1\%) apresentavam impacto muito grave de acordo com o HIT-6. A presença de transtornos mentais comuns foi observada em 82/142 (57,7\%). Houve correlação significativa entre a intensidade da dor referida pelos indivíduos e o escore obtido no HIT-6 $\left(r^{2}=0,2466 ; p<0,0001\right)$, como também entre a intensidade da dor e o escore encontrado no SRQ-20 $\left(r^{2}=0,0887\right.$; $p<0,0003)$. Conclusão: A presença da cefaleia, nos últimos três meses, em policiais militares do Recife, causou impacto negativo na qualidade de vida, com maiores índices de indicativo de morbidade psiquiátrica não psicótica nestes indivíduos.
\end{abstract}

Palavras-chave: Cefaleia; Qualidade de vida; Ocupações; Polícia; Estresse psicológico

\begin{abstract}
Objective: To determine the impact caused by primary headache on quality of life in military policemen of Recife and its relation to the presence of common mental disorders. Methods: This is a transversal study with 211 active policemen, aged between 20 and 50 years (33.0 \pm 7.7 years). The presence of headache was evaluated by using a questionnaire based on ICHD-II, in the last three months. To evaluate the impact of the headache it was used the Headache Impact Test-6. The indicative of probable case of common mental disorder was done by the Self Reporting Questionnaire-20. Pain intensity was measured using a numeric pain rating scale. Results: 142/211 officers (67.3\%) had headache in the last three months. 57/1 42 (40.1\%) were placed in the most severe category in HIT-6. Common mental disorders were observed in 82/1 42 (57.7\%). There was a significant correlation between the intensity of pain reported by the subjects and the score obtained in HIT-6 $\left(r^{2}=0.2466 ; p<0.0001\right)$, as well as between the intensity of pain and the score found in the SRQ-20 $\left(r^{2}=0.0887 ; p<0.0003\right)$. The ten officers who reported to have never had an episode of headache in their lifetime had neither symptoms of mental distress nor were dissatisfied with their work activity. Conclusion: The presence of headache in the military policemen of Recife, in the last three months, caused a negative impact on their quality of life, as well as, a higher indicative of non-psychotic psychiatric morbidity in these individuals.
\end{abstract}

Keywords: Headache; Quality of life; Occupation; Police; Psychological stress 


\section{INTRODUÇÃO}

A cefaleia é um sintoma bastante frequente, sendo considerada um importante problema de saúde pública no Brasil e no mundo devido ao impacto individual e social que causa. ${ }^{(1-4)}$

Qualquer evento que possa alterar a rotina diária do indivíduo de forma positiva ou negativa, através de fonte geradora de estresse, provocará respostas variadas na qualidade de vida. As respostas adaptativas ao estresse são mediadas por características individuais em consequência de uma ação externa, situação ou acontecimento que impõe à pessoa exigências físicas ou psicológicas especiais. ${ }^{(5)}$

Neste contexto, sabe-se que a cefaleia é capaz de gerar impacto direto e indireto na vida dos indivíduos, ${ }^{(6,7)}$ e o estilo de vida sob tensão, causado por fatores psicológicos, socioculturais, ambientais e físicos, contribui para o déficit nas atividades diárias e resulta em diminuição da qualidade de vida. ${ }^{(8,9)}$

Certas atividades laborais podem trazer sérias consequências na qualidade de vida de seus trabaIhadores devido ao ambiente de trabalho no qual estão expostos, fazendo com que se tornem mais susceptíveis a desencadear ou tolerar a cefaleia. ${ }^{(10,11)}$

O papel do policial militar é de fundamental importância para garantir o bem estar social da população.(11) No entanto, para que isso aconteça, os policiais de rua são constantemente expostos ao perigo e à violência no seu dia-a-dia. Tal fato faz com que estejam entre os profissionais que exercem atividades de alto risco, como também os que mais sofrem de estresse. ${ }^{(11,12)}$

Diante do exposto, o estudo tem como objetivo avaliar o impacto da cefaleia primária na qualidade de vida em policiais militares do Recife e sua relação com a presença de transtornos mentais comuns.

\section{MÉTODOS}

Trata-se de um estudo observacional, do tipo transversal. A pesquisa foi realizada entre abril e setembro de 2012. Foram entrevistados 218 policiais militares de rua, homens, atualmente da ativa, da cidade do Recife, Pernambuco, Brasil. A idade dos 211 policiais variou entre 20 e 50 anos (33,0 $\pm 7,7$ anos). Os policiais foram aleatoriamente selecionados nos seus respectivos BataIhões: $11^{\circ}$ (Batalhão 17 de Agosto), $12^{\circ}$ (Batalhão Arraial Novo do Bom Jesus), $13^{\circ}$ (Batalhão Coronel João Nunes), $16^{\circ}$ (Batalhão Frei Caneca) e $19^{\circ}$ (Batalhão André Vidal de Negreiros). Foram excluídos sete questionários devido ao preenchimento incompleto.

Todos os participantes responderam um formulário contendo informações sociodemográficas e ocupacionais, como também questões sobre estresse laboral e presença de cefaleia com suas características. Para avaliar o impacto causado pela cefaleia foi utilizado o questionário Headache Impact Test-6 (HIT-6).(13) A presença de sofrimento mental foi avaliada pelo questionário Self Reporting Questionnaire-20 (SRQ-20)..(14)

O Headache Impact Test-6 (HIT-6) foi desenvolvido por especialistas internacionais em cefaleia, cujo objetivo é medir o impacto da cefaleia na capacidade do indivíduo no trabalho, nos estudos, em casa e em situações sociais. $\bigcirc$ questionário é composto por seis questões que avaliam a gravidade da dor, perda de trabalho, atividades sociais, alterações cognitivas e de humor. $\bigcirc$ impacto da cefaleia é classificado de acordo com o escore em: "pequeno ou nenhum impacto" - naqueles indivíduos que obtiverem um escore de 36 a 49 pontos; "algum impacto" - nos que pontuarem de 50 a 55 pontos; "impacto acentuado" - nos indivíduos com escore entre 56 e 59; "impacto muito grave" - naqueles que alcançarem mais de 60 pontos. ${ }^{(13)}$

O Self Reporting Questionnaire-20 (SRQ-20), desenvolvido pela Organização Mundial de Saúde, é utilizado para mensuração do indicativo de transtornos mentais comuns, que se caracterizam por sintomas não psicóticos, como insônia, fadiga, irritabilidade, esquecimento, dificuldade de concentração e queixas somáticas. Utilizado em estudos brasileiros, especialmente em grupos de trabalhadores, o questionário possui vinte questões e aqueles que obtiverem pontuação maior ou igual a cinco terão indicativo de provável caso de morbidade psiquiátrica não psicótica. $\bigcirc$ instrumento avalia se há algum transtorno, sem, contudo, oferecer um diagnóstico do tipo de transtorno existente..$^{(14)}$

A mensuração da intensidade da dor foi realizada através da escala numérica da dor e seu escore foi classificado em dor leve (1-3), dor moderada (2-7) e dor grave (8-10).

Para efeito de análise estatística, nos questionários HIT-6 e SRQ-20 foram utilizados os dados dos policiais militares com cefaleia nos últimos três meses.

Os dados obtidos na pesquisa são mostrados como média \pm desvio padrão. Utilizamos o teste D'AgostinoPearson para verificar o tipo de distribuição das variáveis a serem estudadas. Para as variáveis que não apresentaram uma distribuição normal utilizamos o teste não 
paramétrico de Mann-Whitney. Na análise das variáveis categóricas aplicamos o teste exato de Fisher. $O$ nível de significância considerado como diferente estatisticamente foi $p<0,05$. Correlação entre variáveis dependentes foi analisada pela regressão linear. Para o processamento e análise dos dados foi o utilizado o programa SPSS versão 13.0 para Windows.

Esta pesquisa foi aprovada pelo Comitê de Ética em Pesquisa do Hospital Agamenon Magalhães (HAM), no mês de novembro de 2011 , cuja numeração é 232; CAAE 0206.0.236.000-11.

\section{RESULTADOS}

No estudo, $211 / 218(96,8 \%)$ policiais militares preencheram corretamente os questionários. Não houve diferença na média de idade entre os batalhões estudados, $p=0,116$. Em relação à presença de cefaleia, 201/211 (95,3\%) referiram ter apresentado algum episódio ao longo da vida, 186/21 1 (88,2\%) no último ano, 142/21 1 (67,3\%) nos últimos três meses e 91/211 $(43,1 \%)$ apresentaram cefaleia na última semana. E ainda, 10/211 (4,7\%) policiais relataram que nunca tiveram cefaleia.

A Tabela 1 mostra as características sociodemográficas e ocupacionais dos 211 participantes. $\bigcirc$ fator remunerativo $[186 / 211(88,2 \%)]$, seguido do trânsito intenso [181/211 (85,2\%)] e da temperatura elevada $[178 / 211(84,4 \%)]$ foram os fatores estressantes mais frequentemente relatados pelos participantes.

Quantificando a dor de acordo com a escala numérica da dor, houve diferença significativa entre os policiais que se queixaram de cefaleia nos últimos três meses $(5,3 \pm 1,9)$ em relação aos sem cefaleia no mesmo período $(3,9 \pm 1,6) ; p<0,001$. Quando avaliada a intensidade da dor por categoria, observou-se que indivíduos com cefaleia nos últimos três meses apresentam escores mais graves quando comparados aos sem cefaleia neste mesmo período, $(p<0,05)$ (Tabela 2).

Houve correlação positiva entre o escore do HIT-6 e a escala de intensidade da dor $\left(r^{2}=0,2466 ; p<0,0001\right)$. Também ocorreu correlação positiva entre a intensidade da dor e o escore obtido no SRQ-20 $\left(r^{2}=0,0887\right.$; $\mathrm{p}<0,0003$ ) (Figura 1).

Os policiais com queixa de cefaleia nos últimos três meses apresentaram maior impacto na qualidade de vida quando comparados aos sem cefaleia neste mesmo período, $[(56 \pm 8,2)$ com cefaleia e $(47,0 \pm 6,9)$ sem cefaleia]; $p<0,05$. Na Tabela 3, 57/142 (40,1\%) dos
Tabela 1. Características sociodemográficas na amostra de 211 policiais militares (idade em anos $33,0 \pm 7,7$ )

\begin{tabular}{|c|c|c|}
\hline Variáveis & $\mathrm{N}$ & $\%$ \\
\hline \multicolumn{3}{|l|}{ Estado civil } \\
\hline Solteiro & 74 & 35,1 \\
\hline Casado & 127 & 60,2 \\
\hline Divorciado & 8 & 3,8 \\
\hline Viúvo & 1 & 0,5 \\
\hline União estável & 1 & 0,5 \\
\hline \multicolumn{3}{|l|}{ Tempo de serviço } \\
\hline De 1 a 3 anos & 82 & 38,9 \\
\hline De 4 a 6 anos & 48 & 22,7 \\
\hline De 7 a 10 anos & 11 & 5,2 \\
\hline$>10$ anos & 70 & 33,2 \\
\hline \multicolumn{3}{|l|}{ Local de trabalho } \\
\hline $11^{\circ}$ Batalhẫo & 24 & 11,4 \\
\hline $12^{\circ}$ Batalhão & 55 & 26,1 \\
\hline $13^{\circ}$ Batalhão & 41 & 19,4 \\
\hline $16^{\circ}$ Batalhão & 53 & 25,1 \\
\hline $19^{\circ}$ Batalhão & 38 & 18,0 \\
\hline \multicolumn{3}{|l|}{ Turno de trabalho } \\
\hline Diurno & 99 & 46,9 \\
\hline Noturno & 52 & 24,6 \\
\hline Alternado & 60 & 28,4 \\
\hline \multicolumn{3}{|l|}{ Horas extras } \\
\hline Sim & 104 & 49,3 \\
\hline Não & 107 & 50,7 \\
\hline \multicolumn{3}{|l|}{ Trabalha fora da PM } \\
\hline Sim & 30 & 14,2 \\
\hline Não & 181 & 85,8 \\
\hline \multicolumn{3}{|c|}{ Equipamento de proteção individual } \\
\hline Sim & 197 & 93,4 \\
\hline Não & 14 & \\
\hline \multicolumn{3}{|l|}{ Satisfação com trabalho } \\
\hline Muito satisfeito & 14 & 6,6 \\
\hline Satisfeito & 84 & 39,8 \\
\hline Pouco satisfeito & 85 & 40,3 \\
\hline Insatisfeito & 28 & 13,3 \\
\hline \multicolumn{3}{|l|}{ Fatores estressantes no trabalho } \\
\hline Fator remunerativo & 186 & 88,2 \\
\hline Trânsito intenso & 181 & 85,2 \\
\hline Temperatura elevada & 178 & 84,4 \\
\hline Violência & 171 & 81,0 \\
\hline Estresse contínuo & 169 & 80,1 \\
\hline Risco iminente de morte & 164 & 77.7 \\
\hline Privação de sono & 160 & 75,8 \\
\hline Excesso de ruídos & 140 & 66,4 \\
\hline Aumento de demanda exces & & \\
\hline serviços em horas extras & 139 & 65,9 \\
\hline Diversidade de ocorrência & 129 & 61,1 \\
\hline Falta de preparo emocional & 51 & 24,2 \\
\hline \multicolumn{3}{|l|}{ Hábito de fumar } \\
\hline Sim & 12 & 5,7 \\
\hline Não & 199 & 94,3 \\
\hline \multicolumn{3}{|l|}{ Consumo de bebida alcóolica } \\
\hline Sim & 99 & 46,9 \\
\hline Não & 112 & 53,1 \\
\hline \multicolumn{3}{|l|}{ Uso de droga } \\
\hline Sim & 2 & 0,9 \\
\hline Não & 209 & 99,1 \\
\hline \multicolumn{3}{|l|}{ Uso medicamento para cefaleia } \\
\hline Sim & 109 & 54,2 \\
\hline Não & 82 & 40,8 \\
\hline \multicolumn{3}{|l|}{ Realiza atividade física } \\
\hline Sim & 161 & 76,3 \\
\hline Não & 50 & 23.7 \\
\hline
\end{tabular}


Tabela 2. Intensidade da cefaleia categorizada em leve, moderado e grave entre os individuos com e sem cefaleia nos últimos três meses

\begin{tabular}{|c|c|c|c|}
\hline \multirow[b]{2}{*}{ Intensidade da dor } & \multicolumn{2}{|c|}{ Grupos } & \multirow[b]{2}{*}{${ }^{*} p$} \\
\hline & $\begin{array}{c}\text { Com cefaleia } \\
n=142\end{array}$ & $\begin{array}{c}\text { Sem cefaleia } \\
n=59\end{array}$ & \\
\hline 0 - 3 (leve) & $25 / 142(17,6 \%)$ & $28 / 59(47,5 \%)$ & 0,0001 \\
\hline 4 - 7 (moderada) & $94 / 142(66,2 \%)$ & $29 / 59(49,2 \%)$ & 0,0269 \\
\hline $8-10$ (grave) & $23 / 142(16,2 \%)$ & $2 / 59(3,4 \%)$ & 0,0100 \\
\hline
\end{tabular}

* Teste exato de Fisher
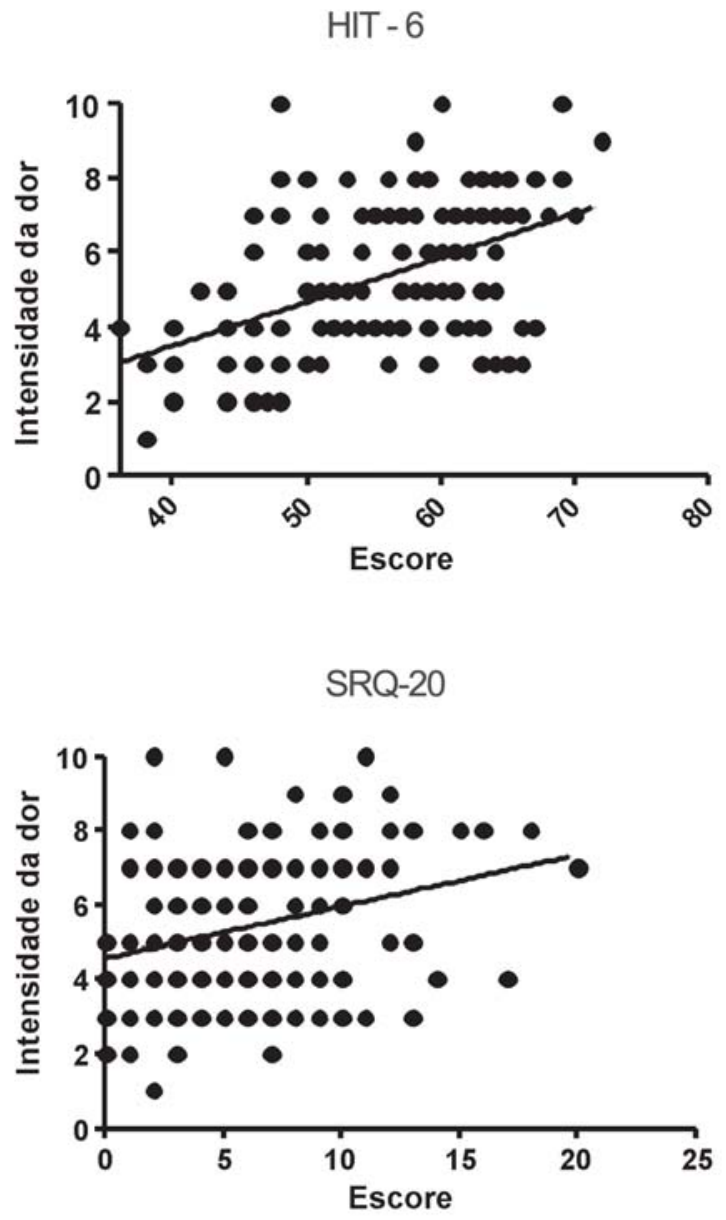

Figura 1. Correlação entre a intensidade da dor (0-10) e o escore obtido no questionário HIT-6 (Headache Impact Test-6, $r^{2}=0,2974$; $p<0,0001$; painel superior) e entre a intensidade da dor (0-10) e o escore obtido no questionário SRQ-20 (Self Reporting Questionnaire-20, $r^{2}=0,0887 ; p=0,0003$; painel inferior) nos 142 indivíduos com cefaleia nos últimos três meses.

policiais com cefaleia nos últimos três meses apresentaram escores elevados, sendo enquadrados na categoria mais grave no HIT-6. No questionário SRQ-20, 93/201 $(46,3 \%)$ policiais apresentam sofrimento mental, dos quais $82 / 93(88,2 \%)$ queixaram-se de cefaleia nos últimos três meses, $p<0,0001$ (Tabela 4).
Tabela 3. Escores obtidos nos questionários HIT-6 (Headache Impact Test-6) e sua comparação entre os indivíduos com e sem cefaleia nos últimos três meses

\begin{tabular}{lccc}
\hline \multirow{2}{*}{$\begin{array}{l}\text { Questionário } \\
\text { HIT-6 }\end{array}$} & \multicolumn{2}{c}{ Grupos } & \multirow{2}{c}{$\mathrm{p}$} \\
\cline { 2 - 3 } & $\begin{array}{c}\text { Com cefaleia } \\
n=142\end{array}$ & $\begin{array}{c}\text { Sem cefaleia } \\
n=59\end{array}$ \\
\hline \multicolumn{1}{c}{$\begin{array}{c}\text { Escore total } \\
\text { Grau de Incapacidade }\end{array}$} & $56 \pm 8,2$ & $47 \pm 6,9$ & $0,0001 \dagger$ \\
I & & & \\
II & $31 / 142(28,8 \%)$ & $42 / 59(71,2 \%)$ & 0,0001 \\
III & $30 / 142(21,1 \%)$ & $11 / 59(18,6 \%)$ & 0,8478 \\
IV & $24 / 142(16,9 \%)$ & $4 / 59(6,8 \%)$ & 0,0734 \\
\hline
\end{tabular}

* Os dados denotam média \pm desvio padrão

† Teste Mann-Whitney

$\ddagger$ Teste exato de Fisher

Tabela 4. Escores obtidos no questionário SRQ -20 (Self Reporting Questionnaire-20) e sua comparação entre os indivíduos com e sem cefaleia nos últimos três meses.

\begin{tabular}{|c|c|c|c|}
\hline \multirow{2}{*}{$\begin{array}{l}\text { Questionário } \\
\text { SRQ-20 }\end{array}$} & \multicolumn{2}{|c|}{ Grupos } & \multirow[b]{2}{*}{${ }^{*} p$} \\
\hline & $\begin{array}{c}\text { Com cefaleia } \\
n=142\end{array}$ & $\begin{array}{c}\text { Sem cefaleia } \\
n=59\end{array}$ & \\
\hline $\begin{array}{l}\text { Com sofrimento } \\
\text { mental }\end{array}$ & $82 / 142(57,7 \%)$ & $11 / 59(18,6 \%)$ & \multirow[b]{2}{*}{0,0001} \\
\hline $\begin{array}{l}\text { Sem sofrimento } \\
\text { mental }\end{array}$ & $60 / 142(42,3 \%)$ & $48 / 59(81,4 \%)$ & \\
\hline
\end{tabular}

* Teste exato de Fisher

Na amostra total, 10/21 1 (4,7\%) afirmaram nunca ter sofrido de algum episódio de cefaleia ao longo da vida. Os dez policiais apresentaram escores abaixo de cinco pontos no questionário SRQ-20, mostrando que eles não apresentam sintomas de transtornos mentais comuns. Quanto à satisfação com o trabalho, foi observado que $1 / 10(10 \%)$ estava muito satisfeito, $8 / 10$ (80\%) estavam satisfeitos e apenas $1 / 10(10 \%)$ estava pouco satisfeito.

\section{DISCUSSÃO}

Os efeitos prejudiciais da cefaleia na capacidade funcional e qualidade de vida estão bem estabelecidos na literatura e a avaliação da incapacidade causada pela cefaleia pode facilitar a tomada de decisões de tratamento adequado, ${ }^{(15-17)}$ que pode ser iniciado de forma aguda ou preventiva. ${ }^{(18)}$

Neste estudo destacou-se a investigação do impacto da cefaleia na qualidade de vida de policiais militares do Recife, atuantes nas ruas e constantemente vivenciando situações de estresse físico e psicológico durante sua atividade laboral. 
O impacto na qualidade de vida gera custos diretos e indiretos. Os custos diretos englobam gastos em assistência médica, ambulatorial e medicamentosa. ${ }^{(19)}$ Estes dados corroboram os achados no presente estudo, pois observou-se que a maioria dos policiais com cefaleia nos últimos três meses [76/142 (53,5\%)] fazia uso de remédio para cefaleia e neste mesmo grupo 82/142 (57,7\%) houve maior presença de transtorno mental comum. Atualmente sabe-se que o uso excessivo de medicações para cefaleia, associado às alterações comportamentais e psicológicas (ansiedade, depressão e distúrbios do sono, bem como anormalidades nos testes de avaliação psicológica), está associado à cronificação da cefaleia. ${ }^{(20)}$

Os custos indiretos estão relacionados à diminuição na produtividade do trabalho, ${ }^{(19,21)}$ que no estudo pode ser visto através da pouca satisfação ou insatisfação dos policiais com o trabalho, onde 12/23 policiais que estavam pouco satisfeitos $(52,2 \%)$ atribuíram maior escore grave(i. e. 8-10) na escala numérica da dor.

A intensidade da dor observada no grupo com cefaleia nos últimos três meses obteve um de escore de $5,3( \pm 1,9)$, o qual pode ter ocorrido devido ao fato dos homens apresentarem menos queixa de dor que as mulheres. Há relato na literatura que mostra a diferença desse escore entre gêneros. Nesse mesmo estudo, a prevalência de condições dolorosas, especialmente a cefaleia, foi maior e com dor mais grave nas mulheres, que pode estar relacionada às flutuações cíclicas de estrogênio e progesterona. ${ }^{(22)}$

grupo de policiais com cefaleia nos últimos três meses obteve um resultado de maior impacto na qualidade de vida dos indivíduos que apresentaram maior escore na intensidade da dor de cabeça, coincidindo com estudos prévios que mostram influência direta da intensidade da dor na qualidade de vida profissional, por gerar incapacidade nos indivíduos que a apresentam. ${ }^{23,24)}$

É importante considerar a organização do trabalho a que os funcionários estão submetidos, pois quando certas situações são apresentadas como natural, existe a desconsideração da organização do trabalho como fator relevante na presença de sofrimento.(25)

Existe na literatura uma demonstração de que uma resposta psicológica à cefaleia está associada à diminuição na qualidade de vida, independentemente das características da dor de cabeça e de outras variáveis demográficas e psicológicas. ${ }^{(26)}$

Respostas do estresse são medidas por características individuais que podem ser de natureza cognitiva, comportamental, afetiva ou fisiológica, englobando estados de apatia, insatisfação, fadiga e ansiedade, podendo ainda gerar distúrbios psicossomáticos de maior gravidade, ${ }^{(5)}$ evidenciando que tanto a cefaleia quanto os transtornos mentais podem coexistir no mesmo indivíduo. ${ }^{(27)}$ Indo de acordo com o que foi dito, no presente estudo observou-se que nos policiais com presença de transtornos mentais comuns houve uma maior pontuação na intensidade da cefaleia.

Os policiais que nunca apresentaram cefaleia na vida também não sofriam de transtornos mentais comuns, nem estavam insatisfeitos com o trabalho. Esse fato poderia sugerir que esses indivíduos se adaptam com mais facilidade a situações de estresse, como visto em estudo anterior, o qual relata que a presença de agentes estressores impõe exigências físicas ou psicológicas especiais às pessoas que são capazes de executar respostas adaptativas ao estresse. . $^{(5,28)}$

De acordo com o que foi visto no presente estudo, a presença de fatores estressantes nas atividades laborais estão relacionadas com o prejuízo na qualidade de vida dos policiais militares com cefaleia. Dessa forma, vale ressaltar a importância de um acompanhamento multidisciplinar para o tratamento, como também trabalhos de prevenção da cefaleia para uma melhora na promoção e proteção da qualidade de vida desses profissionais.

\section{CONCLUSÃO}

A presença da cefaleia, nos últimos três meses, em policiais militares do Recife, causou impacto negativo na qualidade de vida, com maiores índices indicativo de morbidade psiquiátrica não psicótica nestes indivíduos.

\section{REFERÊNCIAS}

1. Queiroz LP, Peres MFP, Kowacs F, Piovesan EJ, Ciciarelli MC, Souza JA, Zukerman E. Um estudo epidemiológico nacional da cefaleia no Brasil. Migrâneas cefaleias. 2008; 11 (3):190-6.

2. Tsuji SR, Carvalho DS. Aspectos psíquicos das cefaleias primárias. Rev. Neurociências. 2002;10(3):129-36.

3. Lipton RB, Bigal ME. Epidemiology of migraine in Latin America: an editorial. Headache. 2005;45:103-4.

4. Rasmussen BK. Epidemiology of headache. Cephalalgia. $2001 ; 21: 774-7$.

5. Bernardi MT, Bussadori SK, Fernandes KPS, Biasotto-Gonzalez DA. Correlação entre estresse e cefaleia tensional. Fisioter. Mov. 2008;21(1):87-93. 
6. Pinto BFB, Santos A, Oliveira CMG, Silva MAJ, Costa BY, Carvalho MC, et al. Prevalência e impacto da cefaleia nos funcionários de uma empresa: avaliação com a colaboração da Associação de Trabalhadores. Migrâneas cefaleias. 2008;11 (4): 229-32.

7. Bigal ME, Speciali JG. Classificação, epidemiologia e impacto das cefaleias crônicas diárias. Einstein. 2004 ;2 (Supl 1): 1 -4.

8. Berg J. Economic evidence in migraine and other headache: a review. Eur J Health Econ. 2004;5 (Supl 1):S543-S554.

9. Belam J, Kernick D, Kline F, et al. A qualitative study of migraine involving patient researchers. Br J Gen Pract. 2005;55: 87-93.

10. Farias SMC, Teixeira OLC, Moreira W, Oliveira MAF, Pereira $\mathrm{MO}$. Caracterização dos sintomas físicos de estresse na equipe de pronto atendimento. Rev Esc Enferm USP. 2011 ; 45(3): 722-9.

11. Patterson GT, Chung IW, Swan PG. The effects of stress management interventions among police officers and recruits. Campbell Systematic Reviews. 2012;7:1-53.

12. Costa M, Accioly Jr H, Oliveira J, Maia E. Estresse: diagnóstico dos policiais militares em uma cidade brasileira. Rev Panam Salud Publica. 2007;21 (4):217-22.

13. The Headache Impact Test (HIT) < www.amlhealthy.com.>

14. Santosa KOB, Araújo TM, Pinho PS, Silva ACC. Avaliação de um instrumento de mensuração de morbidade psíquica: estudo de validação do self-reporting questionnaire (SRQ-20). Rev Baiana Saude Publica Miolo. 2010;34(3):544-60.

15. Shin HE, Park JW, Kim YI, Lee KS. Headache Impact Test-6 (HIT6) scores for migraine patients: their relation to disability as measured from a headache diary. J Clin Neurol. 2008;4:15863.

16. Terwindt GM, Ferrari MD, Tijhuis M, Groenen SM, Picavet HS, Launer LJ. The impact of migraine on quality of life in the general population: the GEM study. Neurology. 2000;55:624-9.

17. Lipton RB, Liberman JN, Kolodner KB, Bigal ME, Dowson A, Stewart WF. Migraine headache disability and health-related quality of-life: a population-based case-control study from England. Cephalalgia. 2003;23:441-50.

18. Peres MFP, Amado DK, Gonçalves AL, Ribeiro R, Pagura JR, Queiroz LP. The need for preventive therapy in primary headaches. Headache Medicine. 2011 ;2(2):46-9.

19. Oliveira DA, Brito JKC, Souza CMS, Cruz CKR, Silva LC, Siqueira $G R$, et al. Cefaleia do tipo tensional e migrânea em funcionários de uma instituição de ensino superior: grau de incapacidade. Headache Medicine. 2011 ;2(2):61-5.

20. Evers S, Jensen R. Treatment of medication overuse headache guideline of the EFNS headache panel. European Journal of Neurology. $2011 ; 18: 115-2$

21. Lambert J, Carides GW, Meloche JP, Gerth WC, Marentette MA. Impact of migraine symptoms on health care use and work loss in Canada in patients randomly assigned in a phase III clinical trial. Can J Clin Pharmacol. 2002;9:158-64.

22. Palmeira CCA, Ashmawi HA, Posso IP. Sexo e percepção da dor e analgesia. Rev Bras Anestesiol. 201 1;61 (6).

23. Sauro KM, Rose MS, Becker WJ, Christie SN, Giammarco R, Mackie GF et al. HIT-6 and MIDAS as measures of headache disability in headache referral population. Headache. 2010; 50:383-95.

Headache Medicine, v.4, n.4, p.96-101, Oct./Nov./Dec. 2013
24. Magnusson JE, Becker WJ. Migraine frequency and intensity: Relationship with disability and psychological factors. Headache. 2003;43: 1049-59.

25. Louzada RCR, Silva Filho JF. Formação do pesquisador e sofrimento mental: um estudo de caso. Psicologia em Estudo. 2005; 10(3):451-61.

26. Holroyd KA, Drew JB, Cottrell CK, Romanek KM, Heh V. Impaired functioning and quality of life in severe migraine: the role of catastrophizing and associated symptoms. Cephalalgia. 2007; 27(10):1156-65.

27. Jette N, Patten S, Williams J, Becker W, Wiebe S. Comorbidity of migraine and psychiatric disorders - a national population-based study. Headache. 2008;48:501-16.

28. Marco PF, Cítero VA, Moraes E, Martins LAN. O impacto do trabalho em saúde mental: transtornos psiquiátricos menores, qualidade de vida e satisfação profissional. J Bras Psiquiatr. 2008;57(3):178-83

Correspondência

Daniella Araúio de Oliveira

Av. Jorn. Anibal Fernandes, s/n, Cidade Universitária, Recife, PE, Brasil, 50740-560. Fone:(55-81) 21268937, Fax: (55-81) 21268491 email:sabino_daniella@ig.com.br

Conflito de interesse: sem conflito de interesse Fonte financiadora do projeto: Recursos próprios

Received: Dec 12, 2013

Accepted: Dec 16, 2013 